\title{
An efficient integral equation technique for the analysis of arbitrarily shaped capacitive waveguide circuits
}

\author{
F. D. Quesada Pereira, ${ }^{1}$ P. Vera Castejón, ${ }^{1}$ A. Álvarez Melcón, ${ }^{1}$ B. Gimeno, ${ }^{2}$ \\ and V. E. Boria Esbert ${ }^{3}$ \\ Received 15 June 2010; revised 15 November 2010; accepted 24 January 2011; published 21 April 2011.
}

[1] In this contribution a new and efficient integral equation formulation is presented for the analysis of arbitrarily shaped capacitive waveguide devices. The technique benefits from the symmetry of the structure in order to reduce the dimensions of the problem from three to two dimensions. For the first time, this technique formulates the waveguide capacitive discontinuity problem as a 2-D scattering problem with oblique incidence, combined with an efficient calculation of the parallel plate Green's functions. The numerical method allows the efficient evaluation of the electromagnetic fields inside the analyzed structures. Results for different practical capacitive waveguide devices are successfully compared with commercial software tools for validation of the proposed theory. Finally, a novel low-pass filter implementation based on circular conducting posts has been proposed. The field contour lines in the critical gaps of the new structure are curved due to the use of rounded posts. This could result in improved power handling capabilities with respect to standard corrugated low-pass filters.

Citation: Quesada Pereira, F. D., P. Vera Castejón, A. Álvarez Melcón, B. Gimeno, and V. E. Boria Esbert (2011), An efficient integral equation technique for the analysis of arbitrarily shaped capacitive waveguide circuits, Radio Sci., 46, RS2017, doi:10.1029/2010RS004458.

\section{Introduction}

[2] Capacitive rectangular waveguide structures are typically used for the design of low-pass filters as proposed by Levy [1973], impedance converters [see Young, 1962], and matching networks. In the past, several numerical techniques have been used for computing the electrical response of this kind of devices, such as the finite elements method [see Salazar Palma et al., 1998] employed by widely used commercial software packages like $\mathrm{HFSS}^{\odot}$, modal analysis techniques as described by Guglielmi and Gheri [1994], or the boundary integral resonant mode expansion (BI-RME) used by Arcioni et al. [1996] for the broad band analysis of capacitive microwave devices. Although general purpose finite elements codes can deal properly with $E$ plane (capacitive) structures, their main drawback is the lack of efficiency, since one has to setup a three dimensional model of the circuits. On the other hand, modal analysis as employed by Guglielmi and Gheri [1994] are very efficient for the study of $E$ plane devices composed of canonical rectangular waveguide sections, but cannot easily handle complex geometries such as rounded corners introduced during mechanical manufacturing proce-

\footnotetext{
${ }^{1}$ Information and Signal Theory Department, Technical University of Cartagena, Cartagena, Spain.

${ }^{2}$ Applied Physics Department, University of Valencia, Valencia, Spain. ${ }^{3}$ Communications Department, Technical University of Valencia, Valencia, Spain

Copyright 2011 by the American Geophysical Union. 0048-6604/11/2010RS004458
}

dures, or other useful elements such as conducting rounded posts. This limitation is due to the need of the computation of the modal chart of the basic waveguide sections defining the whole structure.

[3] Moreover, the method of analytical regularization (MAR) [Nosich, 1999] has successfully been employed for the analysis of $E$ plane and $H$ plane rectangular waveguide microwave devices [Kirilenko et al., 1994, 1996; Lyapin et al., 1996]. This technique allows for the analysis of circular posts inside rectangular waveguides following a procedure similar to that proposed by Twersky [1962]. The main advantage of MAR with respect to a standard integral equation technique solved by the method of moments (MOM), is the formulation in terms of Fredholm matrix equations of the second kind which guarantee numerical convergence. However, MAR cannot deal with arbitrarily shaped capacitive geometries, other than circular posts.

[4] This drawback has been overcome by other techniques allowing the efficient evaluation of modal charts in complex geometries, such as the BI-RME method. Alternatively, the analysis of $E$ plane arbitrarily shaped microwave components can also be performed by computing the admittance matrix of the structure with the 2-D BI-RME technique, as presented by Arcioni et al. [1996]. The technique is based on solving an eigenvalue problem where the whole structure is surrounded by an auxiliary rectangular cavity resonator, and the input and output ports are short circuited. The dynamic variation of the fields inside the structure are expanded in terms of the resonant modes of the surrounding cavity used as a reference. Once the eigenvalue problem is solved, the broadband admittance 


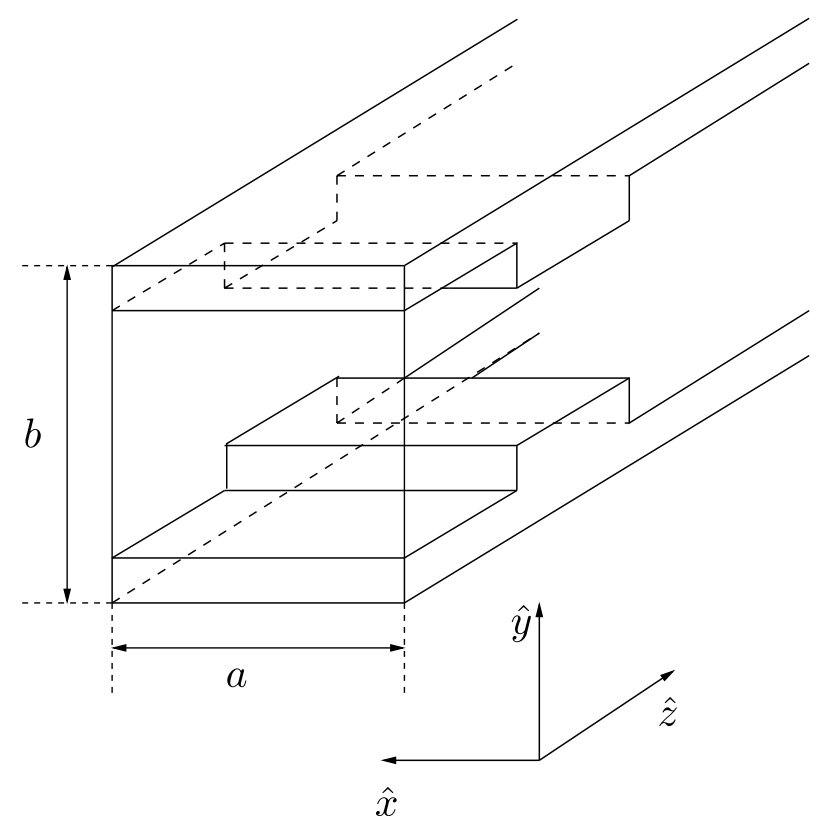

Figure 1. A capacitive step inside a rectangular waveguide of width $a$ and height $b$. The coordinate axis used in this paper is also shown.

parameters of the structure are obtained by opening the input and output ports.

[5] On the other hand, integral equation techniques are very popular for the analysis of useful microwave devices [see Auda and Harrington, 1984; Bunger and Arndt, 2000], and 2-D/3-D scattering problems as described by Harrington [1968] and Rao et al. [1982]. An interesting example is the study of inductive microwave components in rectangular waveguides [see Esteban et al., 2002]. This problem was also solved by Quesada Pereira et al. [2007] and Pérez Soler et al. [2007] using a 2-D integral equation formulation under normal plane wave excitation. In addition, 2-D integral equation formulations with oblique incident angle have been used in general scattering problems, involving both metallic and dielectric objects as described by Peterson et al. [1998] and Rojas [1988]. However, to the authors' knowledge, such methods have never been applied to the study of practical waveguide microwave components. In this paper we solve for the first time the waveguide capacitive discontinuity problem with an oblique incidence integral equation formulation. The technique uses the parallel plate Green's functions of line sources, efficiently computed with the theory previously reported by Quesada Pereira et al. [2007]. Therefore, the calculation of modal charts in complex waveguide structures is not needed for the treatment of complex capacitive waveguide geometries. The proposed technique provides a full-wave analysis of practical capacitive waveguide circuits. Unlike other modal based techniques, it can easily deal with arbitrarily shaped capacitive discontinuities and posts inside rectangular waveguides. The technique is also efficient for the analysis of these devices, since it exploits the symmetry of the structure to reduce the analysis to a 2-D problem. All these reasons make of the technique proposed a very attractive and useful strategy for the design of capacitive waveguide devices.
[6] This contribution presents the basic formulation and the theory underlying the developed integral equation. The technique is demonstrated in three $E$ plane practical waveguide devices, including simulation data from commercial software packages for validation. A novel low-pass filter based on circular capacitive steps is proposed, to show the capabilities of the new technique to deal with complex geometries. The electromagnetic fields distributions are obtained for the two last examples. Results show that in the critical gaps the field contour lines are curved due to the use of rounded posts. This could result into a beneficial effect for power handling capabilities issues with respect to standard corrugated low-pass filters presented by Cameron et al. [2007].

\section{Theory}

[7] The formulation of the proposed method is setup considering a rectangular waveguide where all the discontinuities are invariant along the $x$ axis, as shown in Figure 1. In Figure 1, the relevant dimensions (width $a$ and height $b$ ) of the waveguide are indicated, together with the orientation of the coordinate axes.

[8] The original structure shown in Figure 1 is replaced, after the application of the surface equivalent principle [see Balanis, 1989], by induced electric current densities on the boundaries of the conducting discontinuities. In this way, the rectangular waveguide is reduced to a parallel plate waveguide due to the invariant geometry of the problem along the $x$ axis. After these considerations, an electric field integral equation (EFIE) [see Peterson et al., 1998] is employed for computing the response of the equivalent two-dimensional problem shown in Figure 2.

[9] The excitation of the problem $\left(\vec{E}_{i}, \vec{H}_{i}\right)$ is the dominant mode of the rectangular waveguide $T E_{10}^{z}$. Although the geometry of the capacitive device is invariant along the $x$ axis, this fundamental mode exhibits a half period trigonometric variation of sine-type along the waveguide width, as can be observed from its basic expression:

$$
\vec{E}_{i}=-\frac{A_{10}}{\varepsilon_{0}} \frac{\pi}{a} \sin \left(\frac{\pi}{a} x\right) e^{-j k_{z} z} \hat{y}
$$

where $k_{z}$ is the propagation constant along the waveguide $z$ axis, and $A_{10}$ is an arbitrary amplitude constant [Balanis, 1989]. This variation of the exciting field prevents to formulate the problem as a simple scattering problem of normal incidence contained in the $(y, z)$ plane, as it has been done in the works of Quesada Pereira et al. [2007], Auda and Harrington [1984], Conciauro et al. [1996], and Esteban et al. [2002].

[10] Fortunately, the previous variation of the field along the $x$ axis is known and does not change due to the symmetry of the problem. After applying the Euler's formula to equation (1), the incident electric field can be considered as that produced by two plane waves with an oblique incident angle with respect to the $x$ axis $\left(\vec{k}_{1}=-\frac{\pi}{a} \hat{x}+k_{z} \hat{z}\right.$ and $\vec{k}_{2}=\frac{\pi}{a} \hat{x}+$ $k_{z} \hat{z}$, see Figure 3):

$$
\vec{E}^{i}=-\frac{A_{10}}{\varepsilon_{0}} \frac{\pi}{a}\left(\frac{e^{j \frac{\pi}{a} x}-e^{-j \frac{\pi}{a} x}}{2 j}\right) e^{-j k_{z} z \hat{y}}
$$




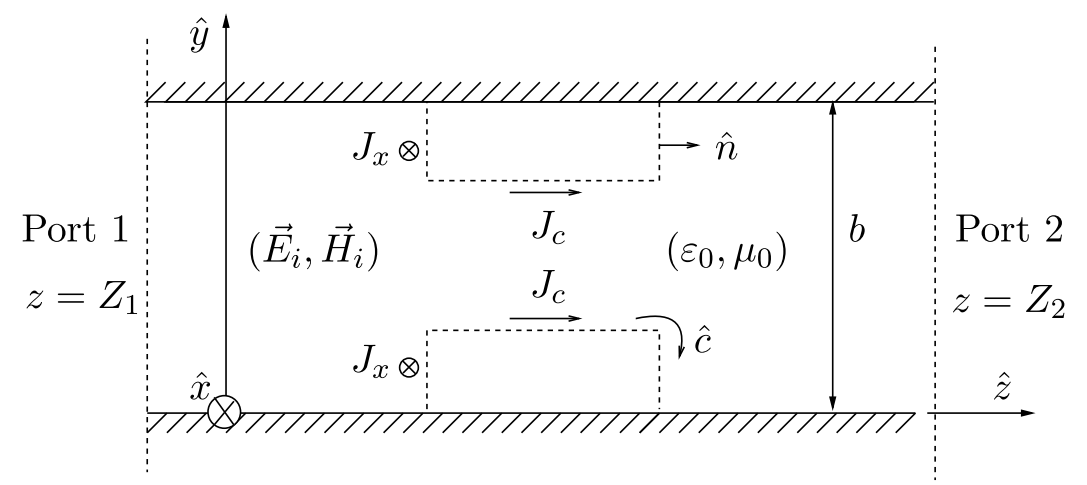

Figure 2. Equivalent parallel plate waveguide problem considered for the evaluation of the electromagnetic response of the device. The constitutive parameters of the medium inside the waveguide are the same as those corresponding to vacuum $\left(\varepsilon_{0}, \mu_{0}\right)$. The electromagnetic fields exciting the structure $\left(\vec{E}_{i}, \vec{H}_{i}\right)$ correspond to the fundamental mode of the rectangular waveguide $T E_{10}^{z} ;(\hat{c})$ is a unit vector tangent to the contour, whereas $(\hat{n})$ is the normal unit vector. The location of the input $\left(z=Z_{1}\right)$ and output $\left(z=Z_{2}\right)$ ports is also represented in the plot.

[11] This decomposition of the exciting field in two plane waves, which are propagating at an oblique angle with respect to the $x$ axis, allows to formulate the problem as a 2-D scattering problem of oblique incidence. Following this theory, the electromagnetic fields scattered by the conducting capacitive discontinuities inside the rectangular waveguide can be computed as the sum of the contributions of the two previous plane waves. A very efficient way for solving this problem is based on writing the EFIE in the dual spatialfrequency domain (spectral variable $k_{x}= \pm \frac{\pi}{a}$ ) [Peterson et al., 1998], resulting:

$$
\begin{gathered}
\hat{n} \times\left[\tilde{\vec{E}}^{i}+\tilde{\vec{E}}^{s}\right]=0 ; \quad \text { on the metallic contour }(c) \\
\hat{n} \times\left[\overrightarrow{\vec{E}}^{i}\right]=\hat{n} \times\left[j \omega \tilde{\vec{A}}+\tilde{\nabla} \Phi_{e}\right] ; \text { on the metallic contour }(c) \\
\tilde{\nabla}=\left(j k_{x} \hat{x}+\frac{d}{d c} \hat{c}\right)
\end{gathered}
$$

where a mixed potential representation of the electric field is assumed, and the $(\sim)$ denotes Fourier transformation with respect to the $x$ coordinate. Using the Green's functions formalism, the explicit form of the integral equation is written as

$$
\begin{aligned}
\left.\tilde{\vec{E}}^{i}(c)\right|_{\tan }= & j \omega \int_{c^{\prime}} \overline{\bar{G}}_{A}\left(\vec{\rho}, \vec{\rho}^{\prime}\right) \cdot\left[\tilde{J}_{x}\left(c^{\prime}\right) \hat{x}+\tilde{J}_{c}\left(c^{\prime}\right) \hat{c}^{\prime}\right] d c^{\prime} \\
& +\left(j k_{x} \hat{x}+\frac{d}{d c} \hat{c}\right) \int_{c^{\prime}} \frac{\left[j k_{x} \tilde{J}_{x}\left(c^{\prime}\right)+\frac{d \tilde{J}_{c}\left(c^{\prime}\right)}{d c^{\prime}}\right]}{-j \omega} \\
& \left.\cdot G_{V}\left(\vec{\rho}, \vec{\rho}^{\prime}\right) d c^{\prime}\right|_{\tan }
\end{aligned}
$$$$
\vec{\rho}=y \hat{y}+z \hat{z}
$$

[12] In equations (4a) and (4b), all the relevant magnitudes are written in a mixed spatial-spectral domain (spatial variables of the contour $(c)$ in the $(y, z)$ plane and the spectral variable $k_{x}$ ).

[13] Another important difference with respect to an inductive problem [see Quesada Pereira et al., 2007; Auda and Harrington, 1984; Esteban et al., 2002], is that the unknown induced electric current $\vec{J}\left(c^{\prime}\right)=\tilde{J}_{x}\left(c^{\prime}\right) \hat{x}+\tilde{J}_{c}\left(c^{\prime}\right) \hat{c}^{\prime}$ presents two components, one along the longitudinal $x$ axis, and the other along the contour $(c)$ of the capacitive problem. On the other hand, $\bar{G}_{A}\left(\vec{\rho}, \vec{\rho}^{\prime}\right)$ is a diagonal dyadic Green's function corresponding to the magnetic vector potential, whereas $G_{V}\left(\vec{\rho}, \vec{\rho}^{\prime}\right)$ is the electric scalar potential Green's function. The mathematical forms of these Green's functions for the geometry under consideration are described in section 2.1.

[14] Finally, the integral equation (equations (3a)-(3c)) has been solved by means of the method of moments expanding

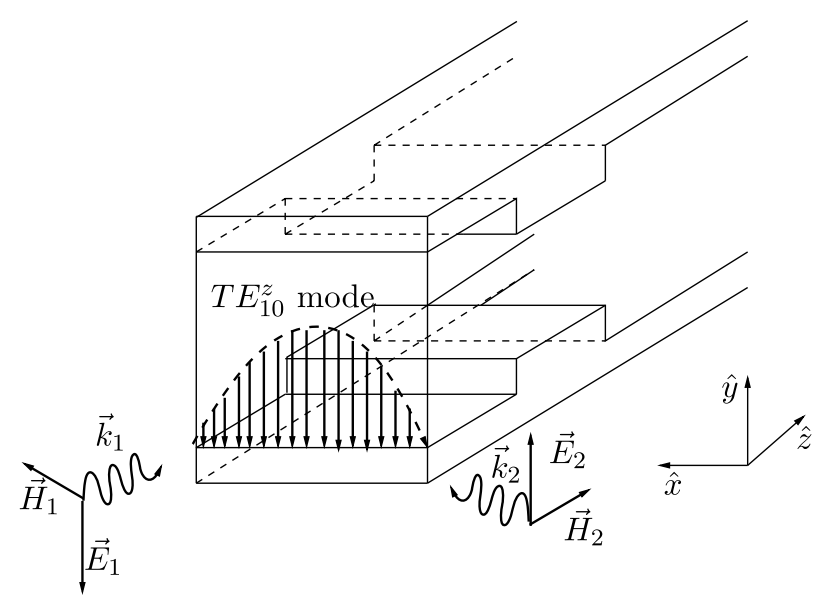

Figure 3. The excitation of the problem, $T E_{10}^{z}$ mode, is the sum of two different plane waves. The propagation constants of the plane waves are $\vec{k}_{1}=-\frac{\pi}{a} \hat{x}+k_{z} \hat{z}$ and $\vec{k}_{2}=\frac{\pi}{a} \hat{x}+k_{z} \hat{z}$. 
the unknown electric current with triangular subsectional basis functions, and using the same set of functions for testing (Galerkin procedure)

$$
\begin{aligned}
\tilde{\vec{J}}\left(c^{\prime}\right)= & \tilde{J}_{x}\left(c^{\prime}\right) \hat{x}+\tilde{J}_{c}\left(c^{\prime}\right) \hat{c}^{\prime}=\sum_{n=1}^{N_{x}} a_{n} f_{n x}\left(c^{\prime}\right) \delta\left(k_{x}-\frac{\pi}{a}\right) \hat{x} \\
& +\sum_{n=1}^{N_{c}} b_{n} f_{n c}\left(c^{\prime}\right) \delta\left(k_{x}-\frac{\pi}{a}\right) \hat{c}^{\prime}=\sum_{n=1}^{N_{x}} a_{n}^{\prime} f_{n x}\left(c^{\prime}\right) \delta\left(k_{x}+\frac{\pi}{a}\right) \hat{x} \\
& +\sum_{n=1}^{N_{c}} b_{n}^{\prime} f_{n c}\left(c^{\prime}\right) \delta\left(k_{x}+\frac{\pi}{a}\right) \hat{c}^{\prime}
\end{aligned}
$$

[15] These functions are defined on the linear segments used in the discretization of the contour of the conducting discontinuities inside the parallel plate waveguide. In the expansion of the currents, $N_{x}$ and $N_{c}$ are the number of subsectional triangular basis functions along the longitudinal $\left(f_{n x}\right)$ and transversal $\left(f_{n c}\right)$ directions, respectively. On the other hand $\delta\left(k_{x} \mp \frac{\pi}{a}\right)$ is a spectral Dirac's delta function defined at the spatial frequencies $\left(k_{x}= \pm \frac{\pi}{a}\right)$ corresponding to the oblique incident angle of the plane waves exciting the rectangular waveguide (see equation (2) and Figure 3). The excitation of the problem can be written in the mixed spatial-frequency domain as:

$$
\tilde{\vec{E}}^{i}=-\frac{A_{10}}{\varepsilon_{0}} \frac{\pi}{a}\left(\frac{\delta\left(k_{x}-\frac{\pi}{a}\right)-\delta\left(k_{x}+\frac{\pi}{a}\right)}{2 j}\right) e^{-j k_{z} z} \hat{y}
$$

[16] The electric current in equations (4a) and (4b) is replaced by its expansion (5). After that, the resulting expression is tested with functions oriented along $x$ axis $\left(f_{m x}\right)$ and along the transveral $(y, z)$ plane $\left(f_{m c}\right)$. Finally, a system of linear equations is obtained and can be written in matrix form for each one of the involved spatial frequencies $\left(k_{x}= \pm \frac{\pi}{a}\right)$, as follows

$$
\left[\begin{array}{ll}
\mathbf{Z}_{\mathbf{m n}}^{\mathbf{x x}} & \mathbf{Z}_{\mathbf{m n}}^{\mathrm{xc}} \\
\mathbf{Z}_{\mathbf{m n}}^{\mathbf{c x}} & \mathbf{Z}_{\mathbf{m n}}^{\mathbf{c}}
\end{array}\right]\left[\begin{array}{c}
\mathbf{a}_{\mathbf{n}}\left(\mathbf{a}_{\mathbf{n}}^{\prime}\right) \\
\mathbf{b}_{\mathbf{n}}\left(\mathbf{b}_{\mathbf{n}}^{\prime}\right)
\end{array}\right]=\left[\begin{array}{c}
\mathbf{0} \\
\mathbf{e}_{\mathbf{m}}
\end{array}\right]
$$

where $\left(\mathbf{a}_{\mathbf{n}}, \mathbf{b}_{\mathbf{n}}\right)$ are the unknown expansion coefficients under plane wave excitation $\left(k_{x}=+\frac{\pi}{a}\right)$, and $\left(\mathbf{a}_{\mathbf{n}}^{\prime}, \mathbf{b}_{\mathbf{n}}^{\prime}\right)$ are the corresponding coefficients under the excitation $\left(k_{x}=-\frac{\pi}{a}\right)$. After some mathematical manipulations, the different submatrices take the form

$$
\begin{gathered}
Z_{m n}^{x x}=j \omega \int_{c_{m}} f_{m x}(c)\left(\int_{c_{n}} G_{A}^{x x}\left(\vec{\rho}, \vec{\rho}^{\prime}\right) f_{n x}\left(c^{\prime}\right) d c^{\prime}\right) d c \\
-\frac{j(\pi / a)^{2}}{\omega} \int_{c_{m}} f_{m x}(c)\left(\int_{c_{n}} G_{V}\left(\vec{\rho}, \vec{\rho}^{\prime}\right) f_{n x}\left(c^{\prime}\right) d c^{\prime}\right) d c \\
Z_{m n}^{x c}=\mp \frac{\pi / a}{\omega} \int_{c_{m}} f_{m x}(c)\left(\int_{c_{n}} G_{V}\left(\vec{\rho}, \vec{\rho}^{\prime}\right) \frac{d f_{n c}\left(c^{\prime}\right)}{d c^{\prime}} d c^{\prime}\right) d c \\
Z_{m n}^{c x}= \pm \frac{\pi / a}{\omega} \int_{c_{m}} \frac{d f_{m c}(c)}{d c}\left(\int_{c_{n}} G_{V}\left(\vec{\rho}, \vec{\rho}^{\prime}\right) f_{n x}\left(c^{\prime}\right) d c^{\prime}\right) d c \\
Z_{m n}^{c c}=\int_{c_{m}} f_{m c}(c)\left[\int_{c^{\prime}}\left(c_{y} c_{y}^{\prime} G_{A}^{y y}\left(\vec{\rho}, \vec{\rho}^{\prime}\right)+c_{z} c_{z}^{\prime} G_{A}^{z z}\left(\vec{\rho}, \vec{\rho}^{\prime}\right)\right) f_{n c}\left(c^{\prime}\right) d c^{\prime}\right] d c \\
-\frac{j}{\omega} \int_{c_{m}} \frac{d f_{m c}(c)}{d c}\left(\int_{c^{\prime}} G_{V}\left(\vec{\rho}, \vec{\rho}^{\prime}\right) \frac{d f_{n c}\left(c^{\prime}\right)}{d c^{\prime}} d c^{\prime}\right) d c \\
e_{m}=\int_{c_{m}} \mp \frac{A_{10}}{\varepsilon_{0}} \frac{\pi}{a} \frac{e^{-j k_{z} z}}{2 j} f_{m c}(c) c_{y} d c
\end{gathered}
$$

The tangent unit vectors to the conducting posts in equations (8a)-(8e) are defined as $\hat{c}=c_{y} \hat{y}+c_{z} \hat{z}$ and $\hat{c}^{\prime}=c_{y}^{\prime} \hat{y}+$ $c_{z}^{\prime} \hat{z}$ for the observation and the source cells, respectively.

[17] Since we are employing the same set of functions for the expansion of the electric current density and for testing, following a Galerkin procedure, the relation $Z_{m n}^{x c}=-Z_{m n}^{c x}$ is satisfied. Moreover, the following relation holds between the current expansion coefficients under the two plane wave excitations:

$$
\begin{aligned}
\mathbf{a}_{\mathbf{n}}^{\prime} & =\mathbf{a}_{\mathbf{n}} \\
\mathbf{b}_{\mathbf{n}}^{\prime} & =-\mathbf{b}_{\mathbf{n}}
\end{aligned}
$$

[18] The previous relations allow to solve the system of linear equation (7) only once for computing the total induced electric current density on the conducting posts. Using these relations, the total electric current in the spatial domain can be expressed as

$$
\begin{aligned}
\vec{J}\left(c^{\prime}\right)= & \sum_{n=1}^{N_{x}} a_{n} f_{n x}\left(c^{\prime}\right) e^{j \frac{\pi}{a} x^{\prime}} \hat{x}+\sum_{n=1}^{N_{x}} a_{n} f_{n x}\left(c^{\prime}\right) e^{-j \frac{\pi}{a} x^{\prime}} \hat{x} \\
& +\sum_{n=1}^{N_{c}} b_{n} f_{n c}\left(c^{\prime}\right) e^{j \frac{\pi}{a} x^{\prime}} \hat{c}^{\prime}-\sum_{n=1}^{N_{c}} b_{n} f_{n c}\left(c^{\prime}\right) e^{-j \frac{\pi}{x^{\prime}} \hat{c}^{\prime}} \\
= & 2 \cos \left(\frac{\pi}{a} x^{\prime}\right) \sum_{n=1}^{N_{x}} a_{n} f_{n x}\left(c^{\prime}\right) \hat{x}+2 j \sin \left(\frac{\pi}{a} x^{\prime}\right) \\
& \cdot \sum_{n=1}^{N_{c}} b_{n} f_{n c}\left(c^{\prime}\right) \hat{c}^{\prime}
\end{aligned}
$$

[19] Once the unknown current has been found, the scattering parameters can directly be computed by evaluating the ratio between the incident and the scattered fields on the ports of the device $\left(z=Z_{1}\right.$ and $z=Z_{2}$ shown in Figure 2), as described by Leviatan et al. [1983]. The computation of the electromagnetic fields is briefly described in section 2.2. It is important to stress that, although the excitation of the capacitive problem is split into two different plane waves, it is only necessary to solve the algebraic problem for one of them, leading to a very efficient formulation.

[20] It can be observed that the proposed technique implements an electric field integral equation (EFIE) for the analysis of capacitive discontinuities inside rectangular waveguides. As it is known, this kind of integral equation may fail at certain frequencies for closed structures. For the practical circuits treated in this paper this is not a problem due to the electrically small size of the conducting posts inside the rectangular waveguides considered. Nevertheless, the presented formulation can be adapted to a combined field integral equation (CFIE) for far out of band analysis. In this case, the posts could become electrically large enough to trigger defect frequencies when using an EFIE. It is known that the CFIE is free from these defect frequencies and yields to correct results in case that numerical problems are encountered.

\subsection{Parallel Plate Green's Functions}

[21] In this section, the parallel plate Green's functions used for solving the capacitive equivalent problem are 
Table 1. Green's Functions Components Needed for Solving the Capacitive Equivalent Problem

\begin{tabular}{cccc}
\hline & $\xi$ & $f_{n}$ & $g_{n}$ \\
\hline$G_{A}^{x x}$ & $\mu_{0}$ & $\sin \left(k_{y} y\right)$ & $\sin \left(k_{y} y^{\prime}\right)$ \\
$G_{A}^{y y}$ & $\mu_{0}$ & $\cos \left(k_{y} y\right)$ & $\cos \left(k_{y} y^{\prime}\right)$ \\
$G_{A}^{z z}$ & $\mu_{0}$ & $\sin \left(k_{y} y\right)$ & $\sin \left(k_{y} y^{\prime}\right)$ \\
$G_{V}$ & $1 / \varepsilon_{0}$ & $\sin \left(k_{y} y\right)$ & $\sin \left(k_{y} y^{\prime}\right)$ \\
\hline
\end{tabular}

summarized. The general expression of these Green's functions in their spectral form is given by:

$$
\begin{gathered}
G_{p p w}\left(z-z^{\prime}, y, y^{\prime}\right)=\frac{\varepsilon_{n} \xi}{b \pi} \sum_{n=0}^{\infty} f_{n}\left(k_{y} y^{\prime}\right) g_{n}\left(k_{y} y\right) \frac{e^{-j k_{z}\left(z-z^{\prime}\right)}}{j k_{z}} \\
k_{z}=\sqrt{k_{0}^{2}-k_{x}^{2}-k_{y}^{2}} ; \quad k_{y}=\frac{n \pi}{b} ; k_{x}=\frac{\pi}{a}
\end{gathered}
$$

where $\varepsilon_{n}=\left\{\begin{array}{ll}1 & n=0 \\ 2 & n \neq 0\end{array}, f_{n}\right.$ and $g_{n}$ are trigonometric functions, and $\xi$ is a constant depending on the constitutive parameters of the medium filling the rectangular waveguide. It is interesting to observe the definition of the longitudinal wavenumber $\left(k_{z}\right)$, which is modified with respect to the inductive case with the fixed oblique incident angle $\left(k_{x}=\pi / a\right)$ used in this formulation. All the relevant definitions for the Green's functions needed in order to solve the capacitive equivalent problem are given in Table 1.

[22] It is worth stressing the low convergent behavior of the series represented in equations (11a) and (11b) when the observation point along the propagation $z$ axis is very close to the source at $z^{\prime}$. For these situations, one has to employ summation acceleration techniques like the Kummer's method used by Quesada Pereira et al. [2007] and Leviatan et al. [1983] for an efficient computation of the Green's functions in the implementation of the integral equation technique.

\subsection{Electromagnetic Fields and Scattering Parameters Evaluation}

[23] The scattered electric field inside the rectangular waveguide is computed by using the following mixed potentials expression:

$$
\vec{E}^{s}(\vec{\rho})=-j \omega \vec{A}(\vec{\rho})-\nabla \Phi_{e}(\vec{\rho})
$$

[24] One can write the previous equation in the spectral domain as follows:

$$
\begin{aligned}
\tilde{\vec{E}}^{s}(\vec{\rho})= & -j \omega \int_{c^{\prime}} \overline{\bar{G}}_{A}\left(\vec{\rho}, \vec{\rho}^{\prime}\right)\left(\tilde{J}_{x}\left(c^{\prime}\right) \hat{x}+\tilde{J}_{c}\left(c^{\prime}\right) \hat{c}^{\prime}\right) d c^{\prime} \\
& -\left(j k_{x} \hat{x}+\frac{d}{d c} \hat{c}\right) \int_{c^{\prime}} G_{V}\left(\vec{\rho}, \vec{\rho}^{\prime}\right)\left[\frac{j k_{x} \tilde{J}_{x}\left(c^{\prime}\right)+\frac{d \tilde{J}_{c}\left(c^{\prime}\right)}{d c^{\prime}}}{-j \omega}\right] d c^{\prime}(13)
\end{aligned}
$$

[25] Due to the dyadic nature of equation (13), we have a different expression for each electric field component. The main component of the electric field will be along the $y$ axis when only the fundamental mode is propagating inside the waveguide. This is because the $x$ component is null, and the $z$ component is only significant in the near-field region surrounding the conducting obstacles. This last component is generated by the capacitive discontinuities, and it will be significant only close to them.

[26] It is important to note that one has to compute the $y$ and $z$ derivatives of the electric scalar potential Green's function $G_{V}\left(\vec{\rho}, \vec{\rho}^{\prime}\right)$ for computing the $y$ and $z$ components of the electric field. Although these Green's function derivatives can be computed directly from its spectral form, presented in section 2.1, the performance of the convergence of the series in equations (11a) and (11b) is worse than the original Green's function, even after the application of the Kummer's acceleration technique. For a better convergence performance, one can switch to a direct computation of the $z$ derivatives in the spatial domain as a series of spatial images [see Quesada Pereira et al., 2007], or to the application of the Ewald acceleration technique as proposed by Capolino et al. [2005] and Quesada Pereira et al. [2006].

[27] The computation of the scattered magnetic field can be carried out by applying a similar procedure to the equation

$$
\vec{H}(\vec{\rho})=\frac{1}{\mu_{0}} \nabla \times \vec{A}(\vec{\rho})
$$

[28] For the capacitive problem the previous expression can be written in the spectral domain as

$$
\begin{aligned}
\tilde{\tilde{H}}(\vec{\rho})= & \frac{1}{\mu_{0}}\left(j k_{x} \hat{x}+\frac{\partial}{\partial y} \hat{y}+\frac{\partial}{\partial z} \hat{z}\right) \\
& \times \int_{c^{\prime}} \overline{\bar{G}}_{A}\left(\vec{\rho}, \vec{\rho}^{\prime}\right)\left(\rho, k_{x_{1}}\right)\left[\tilde{J}_{x}\left(c^{\prime}\right) \hat{x}+\tilde{J}_{c}\left(c^{\prime}\right) \hat{c}^{\prime}\right] d c^{\prime}
\end{aligned}
$$

[29] Taking into account the dyadic nature of equation (15), a different expression is again obtained for each component of the magnetic field.

[30] Finally, to compute the fields in the spatial domain, the above expressions are first evaluated in the spectral domain with the electric current corresponding to each spatial frequency $k_{x}= \pm \frac{\pi}{a}$. After that, the individual results are transformed into the spatial domain and they are summed. Since the electric current component weights $\tilde{J}_{c}\left(c^{\prime}\right)$ and $\tilde{J}_{x}\left(c^{\prime}\right)$ are related in our problem to the harmonics $k_{x}^{1}=\pi / a$ and $k_{x}^{2}=-\pi / a$, as shown in equations (9a) and (9b), similar relationships to (10) can be established for the different electromagnetic fields components in the spatial domain.

\section{Results}

[31] The first example is a low-pass filter with a $12.8 \mathrm{GHz}$ cutoff frequency presented by Levy [1973]. The geometry and dimensions of the filter can be seen in Figure 4. The design is composed of two different sections. The inner section corresponds to a tapered corrugated waveguide lowpass filter derived from a Zolotarev half-stub prototype. The terminal parts are impedance transformers for matching the waveguide impedance of the tapered corrugated filter to the input and output ports of a standard WR-90 waveguide.

[32] The results obtained with the new integral equation technique have been compared again to those provided by the commercial software FEST3D ${ }^{\odot}$ from Aurora Software 


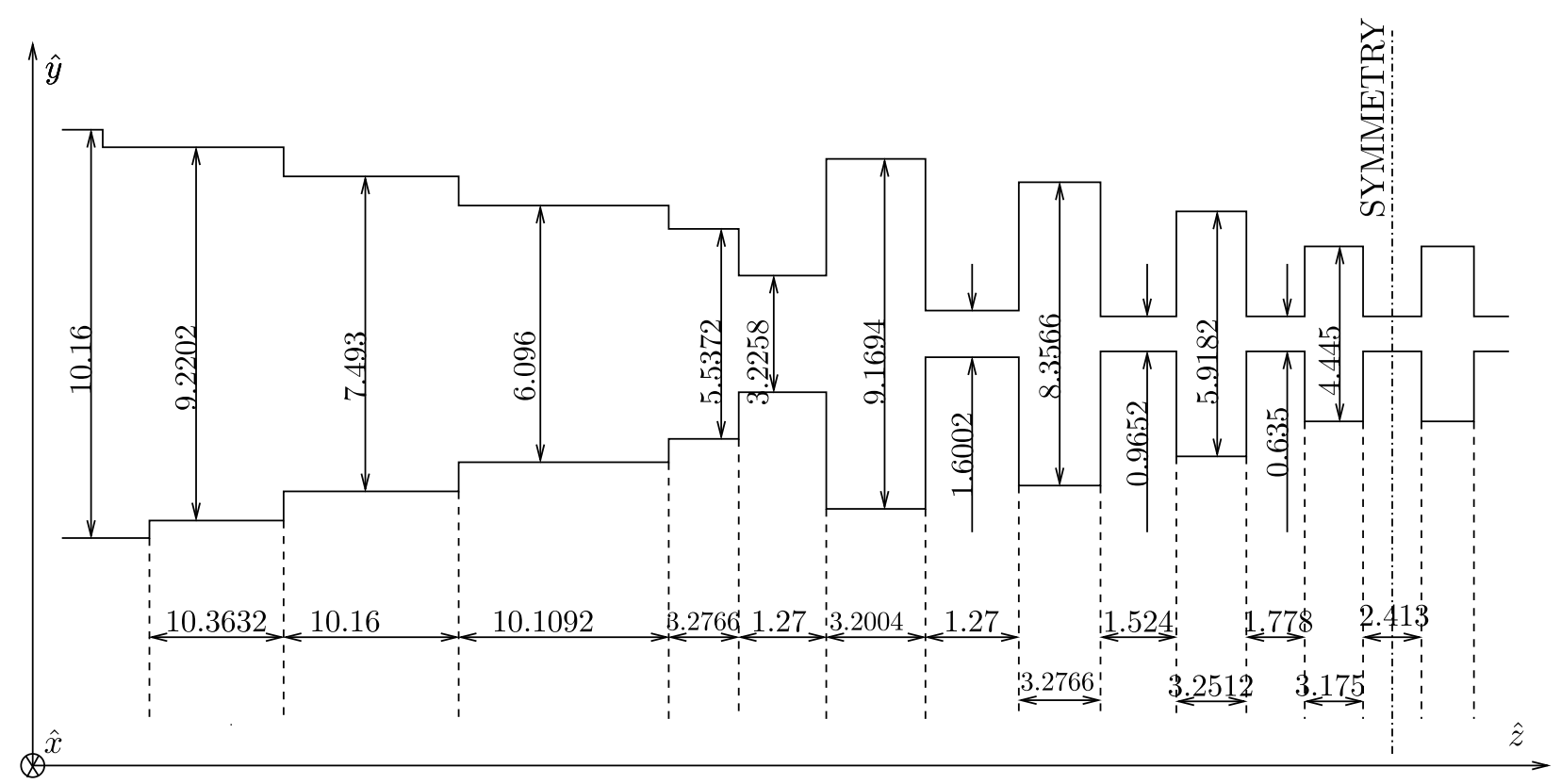

Figure 4. Geometry and dimensions of the low-pass filter presented by Levy [1973]. The filter structure is composed of impedance converters in the input and output ports and a Zolotarev prototype for the inner section. The input and output waveguides are WR-90 $(a=22.86 \mathrm{~mm}, b=10.16 \mathrm{~mm})$.

and Testing, S.L. [2009], which is based on the integral equation technique presented by Álvarez Melcón et al. [1996], showing a very good agreement, as can be observed in Figure 5. The results are also in good agreement with respect to the measured results reported by Levy [1973], thus validating the software tool developed. In this case, only 15 modes have been enough for the Green's functions computation, whereas the number of unknowns for expanding the surface current density has been set to 1200 . The evaluation time has been $0.51 \mathrm{~s}$ per frequency point in a 64 bits computer with a $2.0 \mathrm{GHz}$ clock.

[33] Although we have employed general purpose basis functions for expanding the unknown electric current density on the discontinuities (triangular basis functions), we have also taken care of the fast electromagnetic field variations close to conducting sharp corners. In this case, the electric current is better modeled with a finer mesh density around these corners. We have employed a cosine-like mesh pattern for straight edges forming a corner. This pattern provides a faster convergence of the solution than a uniform mesh, since it concentrates more mesh cells in the proximity of the corners.

[34] The new technique has been employed for the analysis and design of a different low-pass filter implementation. In this case, the filter is composed of different capacitive irises acting as impedance inverters, inside a constant height rectangular waveguide section with dimensions $a=10.68 \mathrm{~mm}$ and $6.0 \mathrm{~mm}$ (see Figure 6). In this design, all waveguide sections are selected of the same characteristic impedance, and the impedance inverters are varied to recover the desired transfer function as described by Cameron et al. [2007]. The results obtained by the new numerical technique have been compared to those provided by the commercial software tool FEST3D ${ }^{\odot}$ from Aurora Software and Testing, S.L. [2009], showing a very good agreement as can be observed in Figure 7. The maximum number of modes used for computing the Green's functions presented in section 2.1 is 10 , whereas 380 basis functions have been enough for characterizing the unknown electric currents, yielding to an accurate response in the frequency range of the analysis. This structure is particularly convenient for the software tool, since all impedance inverters

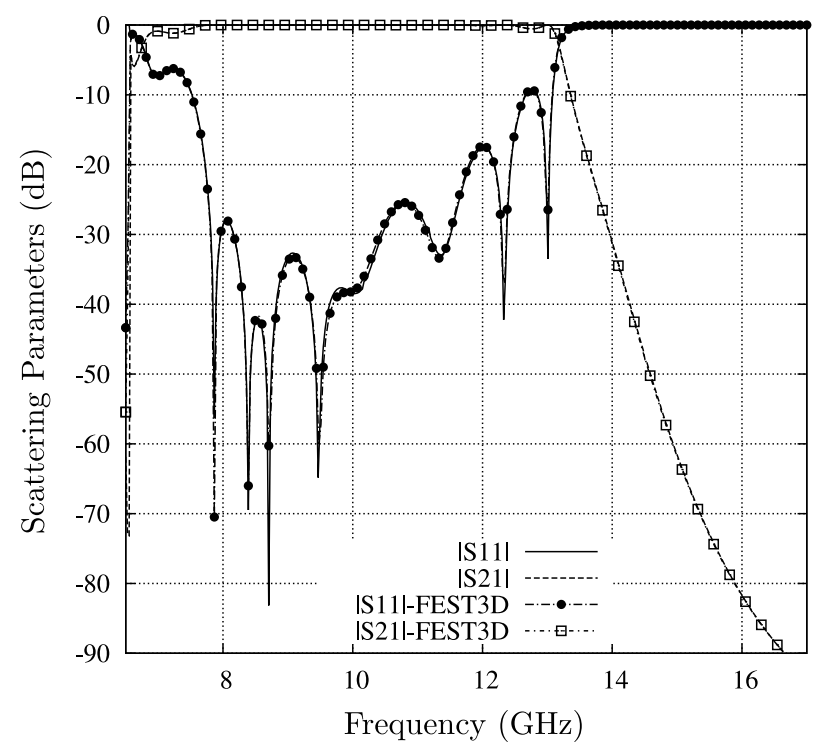

Figure 5. Scattering parameters of the low-pass filter proposed by Levy [1973]. The geometry and dimensions of the filter are shown in Figure 4. The results computed by the new integral equation technique have been compared to those provided by the commercial software tool FEST3D ${ }^{\circ}$ [Aurora Software and Testing, S.L., 2009]. 


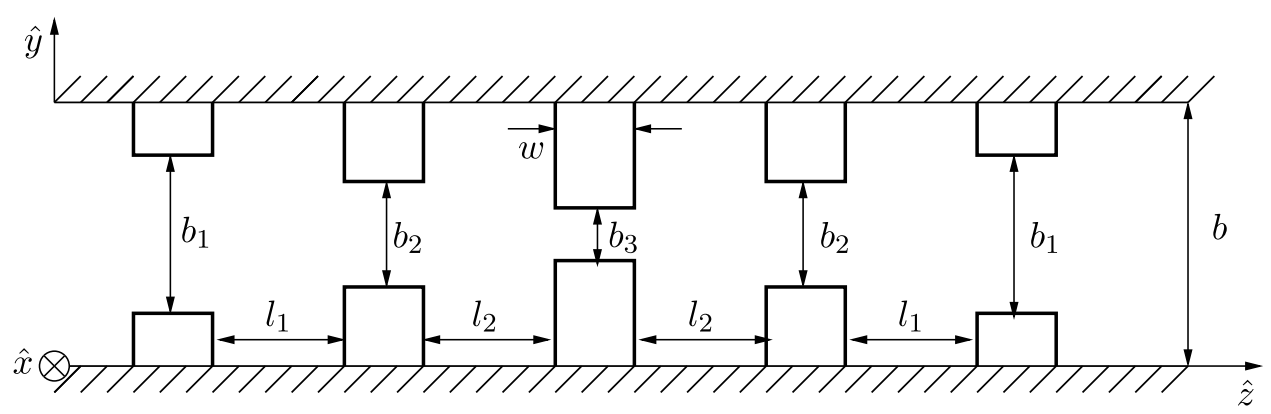

Figure 6. Capacitive low-pass filter composed of rectangular irises inside a rectangular waveguide $(a=$ $10.68 \mathrm{~mm}$ and $b=6.0 \mathrm{~mm}$ ). The dimensions of the filter are: $b_{1}=3.4 \mathrm{~mm}, b_{2}=1.7 \mathrm{~mm}, b_{3}=1.26 \mathrm{~mm}$, $l_{1}=6.55 \mathrm{~mm}, l_{2}=5.0 \mathrm{~mm}$ and $w=4.0 \mathrm{~mm}$.

share a common waveguide section. As a consequence, only the capacitive steps need to be meshed, leading to a reduced number of unknowns. With these considerations, the simulation time is less than $0.2 \mathrm{~s}$ per frequency point in the same computer as used with the previous example.

[35] To show the usefulness of the procedure presented in section 2.2 for the evaluation of the electromagnetic fields inside the capacitive structure, the total electric field inside the rectangular waveguide has been computed at $15 \mathrm{GHz}$ on the $(y, z)$ plane for $x=a / 2$. This frequency is within the passband of the capacitive filter. As can be seen in Figure 8, the highest electric field magnitude is located inside the shortest gap at the center of the structure. This is, therefore, the critical gap of most concern in power handling capability applications. In Figure 8 we can also clearly observe the fringing fields at the corners of the capacitive windows, where they show fast variations. Due to these fast variations we have verified for this example that improved accuracy is obtained when a cosine pattern mesh is used to define the capacitive windows. The field plots shown in Figure 8 are computed with this cosine pattern discretization. Similar considerations can also be applied to the total magnetic field shown in Figure 9.

[36] The next example is a sixth-order capacitive lowpass filter composed of six circular conducting posts inside a constant height rectangular waveguide, with the same dimensions as in the previous design $(a=10.68 \mathrm{~mm}$ and $b=$ $6.0 \mathrm{~mm}$ ) (see Figure 10). To the authors' knowledge, this is the first capacitive low-pass filter design using circular conducting post instead of corrugated rectangular waveguide sections. Results obtained with the new integral equation technique have been compared again to those estimated by the commercial full-wave simulation tool FEST3D (Figure 11). The agreement exhibited by the two results is also good, despite of following very different numerical approaches.

[37] The maximum number of modes used for computing the Green's functions presented in section 2.1 is 10 , whereas 360 basis functions have been enough for characterizing the unknown electric currents, yielding to an accurate response in the studied frequency range. The simulation time is less than $0.18 \mathrm{~s}$ per frequency point in the same computer.

[38] The electromagnetic fields have also been computed for this filter at the same cut plane as done before, and at $15 \mathrm{GHz}$, well inside the passband of the filter. As can be seen in Figure 12 the total electric field is concentrated on the top and bottom areas of the circular conducting posts. The narrower gaps support the highest electric field intensities. However, the contour lines tend to bend due to the curvature of the circular posts. This might be a beneficial effect for power handling capabilities issues. Again, similar considerations can be applied to the magnetic field presented in Figure 13 for completeness.

\section{Conclusions}

[39] A new integral equation technique has been presented for the analysis of arbitrarily shaped capacitive microwave waveguide circuits. For the first time, the scattering parameters of these kind of devices have been computed by formulating a 2-D scattering problem with oblique incident angle. The boundary conditions of the original host waveguide have been taken into account through the use of the

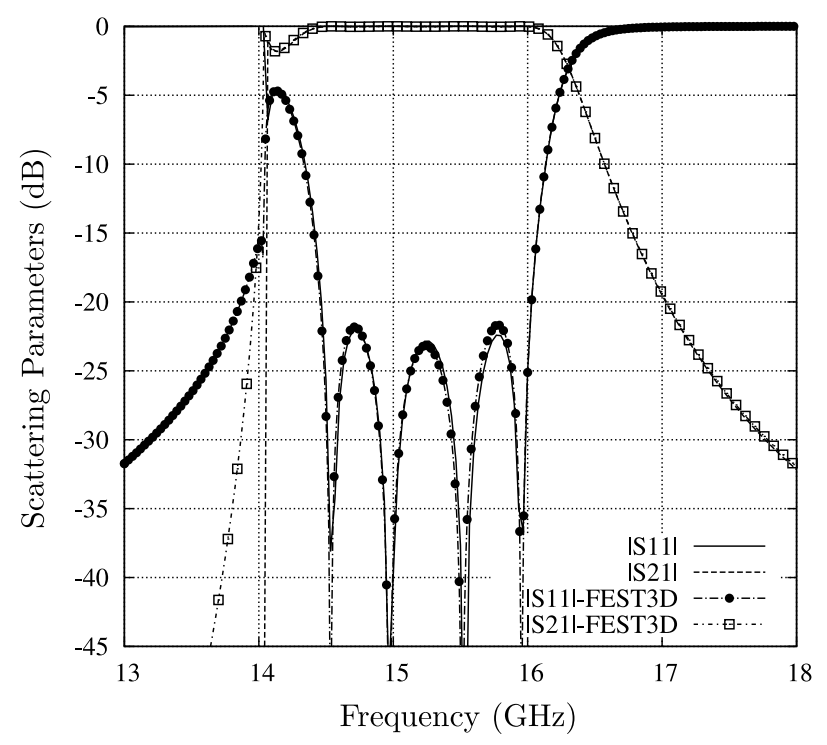

Figure 7. Scattering parameters of the low-pass filter shown in Figure 6. Results computed by the new proposed numerical method have been compared to those data provided by the commercial software package FEST3D ${ }^{\circ}$. 


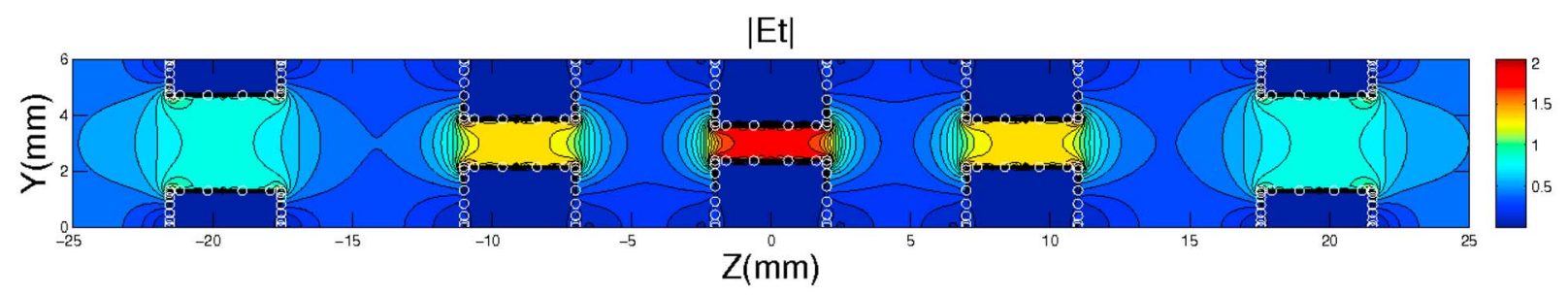

Figure 8. Total electric field magnitude inside the rectangular waveguide at $15 \mathrm{GHz}(x=a / 2)$. The nodes of the mesh employed for the analysis have been also plotted as white circles.

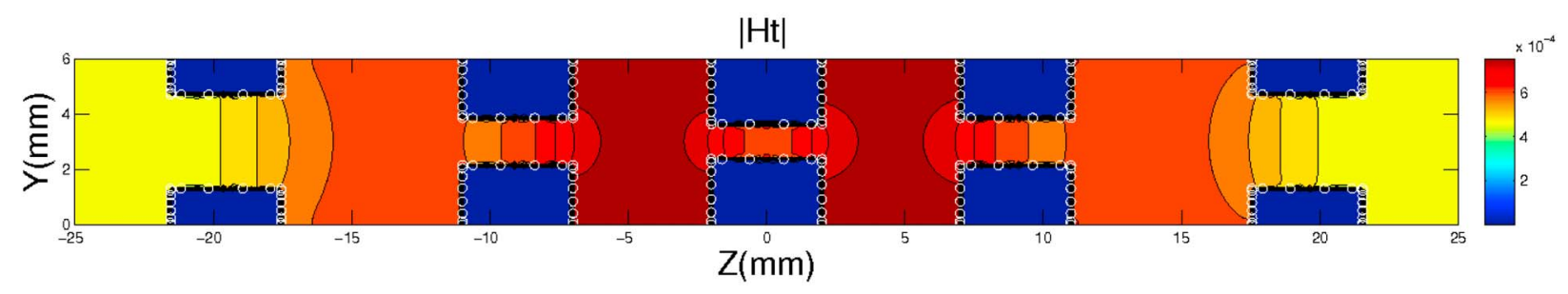

Figure 9. Magnitude of the magnetic field $x$ component inside the rectangular waveguide at $15 \mathrm{GHz}$ ( $x=$ $a / 2)$. The nodes of the mesh employed for the analysis have been also plotted as white circles. 


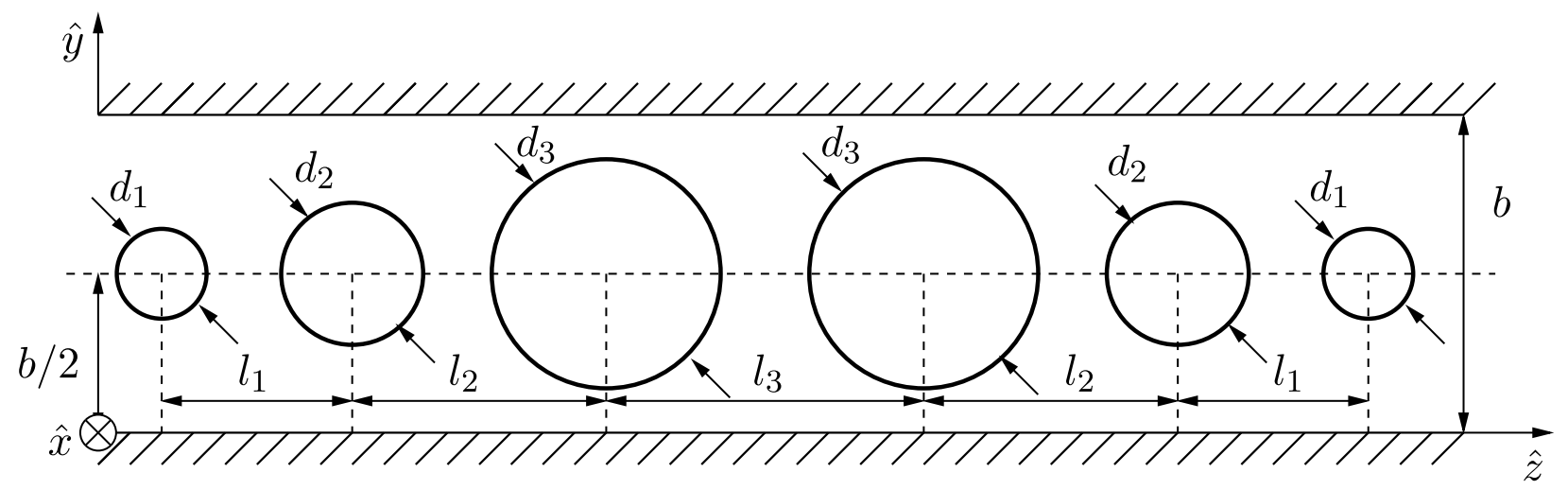

Figure 10. Capacitive low-pass filter composed of six circular conducting posts inside a rectangular waveguide $(a=10.68 \mathrm{~mm}$ and $b=6.0 \mathrm{~mm})$. The dimensions of the structure are: $d_{1}=3.0 \mathrm{~mm}, d_{2}=$ $4.2 \mathrm{~mm}, d_{3}=4.68 \mathrm{~mm}, l_{1}=10.2 \mathrm{~mm}, l_{2}=9.5 \mathrm{~mm}$ and $l_{3}=9.2 \mathrm{~mm}$.

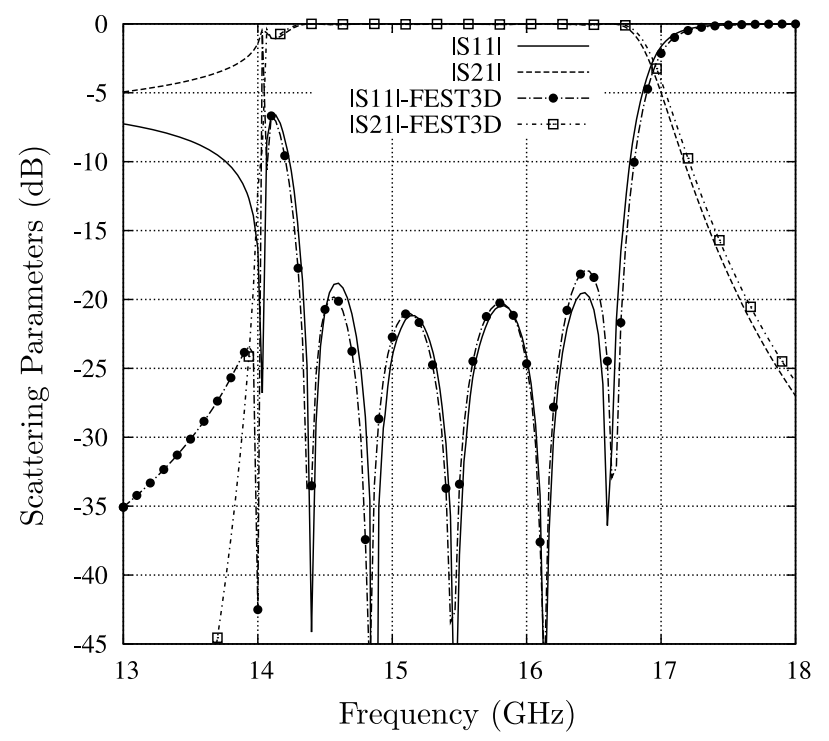

Figure 11. Scattering parameters of the low-pass filter shown in Figure 10. Results computed by the new proposed numerical method have been compared to those data provided by the commercial software package FEST3D ${ }^{\odot}$. 


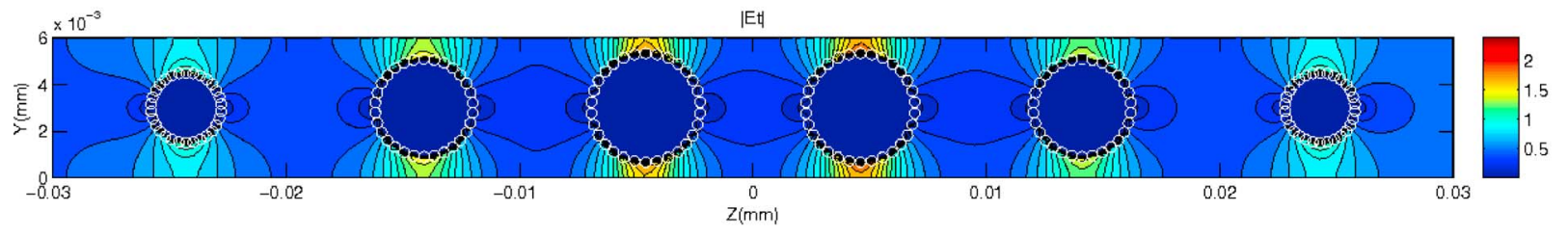

Figure 12. Total electric field magnitude $(\mathrm{V} / \mathrm{m})$ inside the rectangular waveguide at $15 \mathrm{GHz}(x=a / 2)$. The nodes of the mesh employed for the analysis has been also plotted as white circles $\left(A_{10}=\varepsilon_{0} a /(2 j \pi)\right.$ in equation (1)).

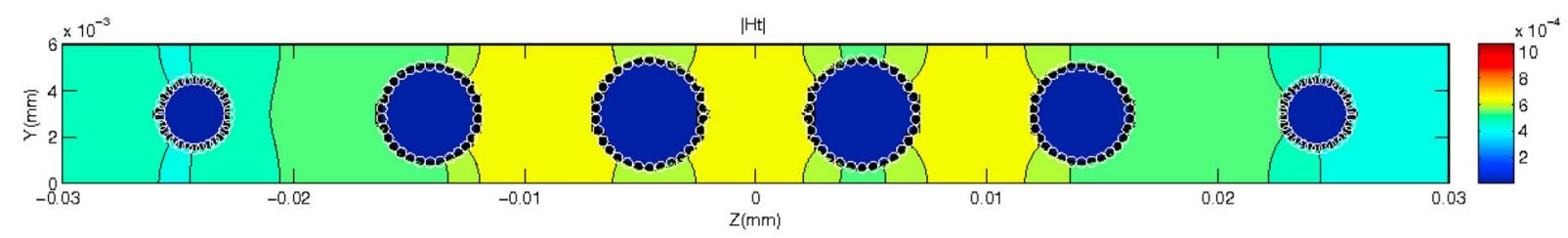

Figure 13. Magnitude of the total magnetic field inside the rectangular waveguide at $15 \mathrm{GHz}(x=a / 2)$. The nodes of the mesh employed for the analysis have been also plotted as white circles $\left(A_{10}=\varepsilon_{0} a /(2 j \pi)\right.$ in equation (1)). 
parallel plate Green's functions of infinite line sources. Results are validated with the analysis of several practical capacitive waveguide devices, such as low-pass filters with different geometries. The technique has also been employed for the efficient evaluation of the electromagnetic fields inside the proposed structures. A new capacitive low-pass filter composed of circular conducting posts has been designed using the software tool. In all cases, simulation results compared to data from commercial software tools and the technical literature have shown the validity and accuracy of the new method.

[40] Acknowledgments. This work has been developed with financial support from SENECA project reference 08833/PI/08, and CICYT project reference TEC2007-67630-C03.

\section{References}

Álvarez Melcón, A., G. Connor, and M. Guglielmi (1996), New simple procedure for the computation of the multimode admittance or impedance matrix of planar waveguide junctions, IEEE Trans. Microwave Theory Tech., 44(3), 413-418.

Arcioni, P., M. Bressan, G. Conciauro, and L. Perregrini (1996), Wideband modelling of arbitrarily shaped E-plane componentes by the boundary integral-resonant mode expansion method, IEEE Trans. Microwave Theory Tech., 44(11), 2083-2092.

Auda, H., and R. F. Harrington (1984), Inductive posts and diaphragms of arbitrary shape and number in a rectangular waveguide, IEEE Trans. Microwave Theory Tech., 32(6), 606-613.

Aurora Software and Testing, S.L. (2009), Full-wave electromagnetic simulation tool 3D v6.0, Valencia, Spain. [Available at http:/www.fest3d. com.]

Balanis, C. A. (1989), Advanced Engineering Electromagnetics, John Wiley, Chichester, U. K.

Bunger, R., and F. Arndt (2000), Moment method analysis of arbitrarily 3D metallic N-port waveguide structures, IEEE Trans. Microwave Theory Tech., 48(4), 531-537.

Cameron, R. J., R. Mansour, and C. M. Kudsia (2007), Microwave Filters for Communication Systems: Fundamentals, Design and Applications, Wiley-Interscience, Chichester, U. K.

Capolino, F., D. R. Wilton, and W. A. Johnson (2005), Efficient computation of the 2-D Green's function for 1-D periodic structures using the Ewald method, IEEE Trans. Antennas Propag., 53(9), 2977-2984.

Conciauro, G., P. Arcioni, M. Bressan, and L. Perregrini (1996), Wideband modeling of arbitrarily shaped $\mathrm{H}$-plane waveguide components by the "boundary integral-resonant mode expansion method", IEEE Trans. Microwave Theory Tech., 44(7), 1057-1066.

Esteban, H., S. Cogollos, V. Boria, A. A. San Blas, and M. Ferrando (2002), A new hybrid mode-matching/numerical method for the analysis of arbitrarily shaped inductive obstacles and discontinuities in rectangular waveguides, IEEE Trans. Microwave Theory Tech., 50(4), 1219-1224.

Guglielmi, M., and G. Gheri (1994), Rigurous multimode equivalent network representation of capacitive steps, IEEE Trans. Microwave Theory Tech., 42(4), 622-628.

Harrington, R. F. (1968), Field Computation by Moment Methods, MacMillan, New York.
Kirilenko, A. A., S. L. Senkevich, V. I. Tkachenko, and B. G. Tysik (1994), Waveguide diplexer and multiplexer design, IEEE Trans. Microwave Theory Tech., 42(7), 1393-1396.

Kirilenko, A. A., L. A. Rud, and V. I. Tkachenko (1996), Semi-inversion method for mathematically accurate analysis of rectangular waveguide $H$ plane angular discontinuities, Radio Sci., 31(5), 1271-1280, doi:10.1029/ 96RS01056.

Leviatan, Y., P. G. Li, A. T. Adams, and J. Perini (1983), Single post inductive obstacle in rectangular waveguide, IEEE Trans. Microwave Theory Tech., 31(10), 806-812.

Levy, R. (1973), Tapered corrugated waveguide low-pass filters, IEEE Trans. Microwave Theory Tech., 21(8), 526-532.

Lyapin, V. P., M. B. Manuilov, and G. P. Sinyavsky (1996), Quasi-analytical method for analysis of multisection waveguide structures with step discontinuities, Radio Sci., 31(6), 1761-1772, doi:10.1029/96RS02558.

Nosich, A. I. (1999), The method of analytical regularization in wavescattering and eigenvalue problems: foundations and review of solutions, IEEE Antennas Propag. Mag., 41(3), 34-39.

Pérez Soler, F. J., F. D. Quesada Pereira, J. Pascual García, D. Cañete Rebenaque, and A. Álvarez Melcón (2007), Efficient integral equation formulation for inductive waveguide components with posts touching the waveguide walls, Radio Sci., 42, RS6002, doi:10.1029/2006RS003591.

Peterson, A. F., S. L. Ray, and R. Mittra (1998), Computational Methods for Electromagnetics, chap. 8, pp. 321-323, IEEE Press, Piscataway, N. J.

Quesada Pereira, F. D., F. J. Pérez Soler, B. Gimeno Martínez, V. E. Boria Esbert, J. Pascual García, J. L. Gómez Tornero, and A. Alvarez Melcón (2006), Efficient analysis tool of inductive passive waveguide components and circuits using a novel spatial domain integral equation formulation, paper presented at 36th European Microwave Conference, Inst. of Electr. and Electron. Eng., Manchester, U. K.

Quesada Pereira, F. D., V. E. Boria Esbert, J. Pascual García, A. Vida Pantaleoni, A. Álvarez Melcón, J. Gómez Tornero, and B. Gimeno Martínez (2007), Efficient analysis of arbitrarily shaped inductive obstacles in rectangular waveguides using a surface integral equation formulation, IEEE Trans. Microwave Theory Tech., 55(4), 715-721.

Rao, S. M., D. R. Wilton, and A. W. Glisson (1982), Electromagnetic scattering by surfaces of arbitrarily shape, IEEE Trans. Antennas Propag., 30(5), 409-418.

Rojas, R. G. (1988), Scattering by an inhomogeneous dielectric/ferrite cylinder of arbitrary cross-section shape, oblique incidence case, IEEE Trans. Antennas Propag., 36(2), 238-246.

Salazar Palma, M., T. K. Sarkar, L. E. G. T. Roy, and A. Djordjevic (1998), Iterative and Self-Adaptive Finite Elements in Electromagnetic Modeling, Artech House, Norwood, Mass.

Twersky, V. (1962), On scattering of waves by the infinite grating of circular cylinders, IRE Trans. Antennas Propag., 10(6), 737-765.

Young, L. (1962), Stepped-impedance transformers and filter prototypes, IEEE Trans. Microwave Theory Tech., 10(5), 339-359.

A. Álvarez Melcón, F. D. Quesada Pereira, and P. Vera Castejón, Information and Signal Theory Department, Technical University of Cartagena, Campus de la Muralla del Mar, Cuartel de Antigones, E-30202 Cartagena, Spain. (fernando.quesada@upct.es)

V. E. Boria Esbert, Communications Department, Technical University of Valencia, Camino de Vera s/n, E-46022 Valencia, Spain.

B. Gimeno, Applied Physics Department, University of Valencia, C. Dr. Moliner 50, Burjassot, E-46100 Valencia, Spain. 